\title{
Premières traces d'anthropisation et évolution des activités agropastorales revelées par l'analyse pollinique des zones humides. Application au sud- Cantal
}

$n^{\circ} 2004 / 137$ et $2006 / 148$

Yannick Miras et Frédéric Surmely

\section{OpenEdition}

Journals

Édition électronique

URL : http://journals.openedition.org/adlfi/4692

ISSN : 2114-0502

Éditeur

Ministère de la culture

Référence électronique

Yannick Miras et Frédéric Surmely, «Premières traces d'anthropisation et évolution des activités agropastorales revelées par l'analyse pollinique des zones humides. Application au sud-Cantal », ADLFI. Archéologie de la France - Informations [En ligne], Auvergne, mis en ligne le 01 mars 2006, consulté le 19 avril 2019. URL : http://journals.openedition.org/adlfi/4692

Ce document a été généré automatiquement le 19 avril 2019

(C) Ministère de la Culture et de la Communication, CNRS 


\title{
Premières traces d'anthropisation et évolution des activités agropastorales revelées par l'analyse pollinique des zones humides. Application au sud-Cantal
} $n^{\circ} 2004 / 137$ et $2006 / 148$

\author{
Yannick Miras et Frédéric Surmely
}

2006 correspond à la dernière année du programme de recherches triennal. L'accent a été mis sur la poursuite des prospections systématiques pédestres et à la finalisation des études paléoenvironnementales (BSR 2003, p. 48 ; BSR 2004, p. 44 ; BSR 2005, p. 50-52).

Du point de la vue de la prospection, la campagne a été orientée sur les zones encore non reconnues (parties hautes des communes de Malbo et Pailherols, est de la commune de Saint-Clément). Par ailleurs, nous avons réexaminé certains secteurs déjà prospectés les années précédentes, ce qui a permis de nouvelles découvertes, notamment en ce qui concerne les vestiges préhistoriques. Cela montre bien le caractère aléatoire des prospections dans ce type de milieu, marqué par une couverture végétale permanente (prairies) et où les découvertes sont conditionnées par la présence de creusements naturels ou anthropiques (travaux d'aménagement agricoles, aménagement de pistes, etc.).

Deux cent soixante-quatorze sites et indices de sites ont été recensés en 2006, ce qui porte les découvertes faites sur trois ans à 506 sites et indices de sites, dont 143 pour la période préhistorique et 256 pour la période médiévale-moderne. Cela confirme bien la grande richesse archéologique de ce secteur de moyenne montagne, dont l'altitude s'étage entre $1100 \mathrm{~m}$ et $1600 \mathrm{~m}$.

Pour la préhistoire, il faut signaler que beaucoup de découvertes préhistoriques se limitent à quelques vestiges lithiques, voire même une pièce isolée. De plus, les sondages pratiqués sur les concentrations de vestiges ont montré l'absence de niveaux en place, ces 
remaniements étant liés à l'action particulièrement forte de l'érosion et à la faible puissance de la couverture sédimentaire superficielle. Malgré cela, il est possible d'avancer des interprétations. Tout d'abord, la répartition des sites n'est pas uniforme, avec des concentrations et au contraire des zones vides, ce qui montre que l'action destructrice de l'érosion n'a pas eu pour effet un transport à grande distance et un étalement général des vestiges. La localisation des sites ne parait pas avoir de lien précis avec des particularités géographiques, telles que point d'eau, axe naturel, en dehors peutêtre des dépressions tourbeuses. De même, la densité de découvertes est aussi importante, voire plus forte dans les secteurs élevés (altitude $>1400 \mathrm{~m}$ ), que dans la partie basse de la zone d'étude.

Le caractère des découvertes empêche la plupart du temps une caractérisation chronologique précise. Le plus ancien site datable se rapporte au Mésolithique (moyen). La période du Mésolithique final-Néolithique ancien est la mieux représentée. Une structure de pierres, reconnue sur la commune de Malbo, pourrait correspondre à un petit coffre mégalithique. Durant l'été, nous avons pratiqué un sondage sur l'indice de site ${ }^{\circ} 426$ (lieu-dit le "Buron des Blats ", commune de Malbo), où des pièces lithiques datables du Mésolithique final avaient été découvertes (BSR 2005, p. 50-52, Yannick Miras). L'opération a montré l'absence de couverture sédimentaire et donc de site en place.

La protohistoire est représentée de façon certaine par 11 indices de sites, dont la datation s'échelonne du Bronze final à La Tène finale (détermination: Patrick Pion). S'y ajoute un nombre important de tertres (152 recensés) dont la caractérisation demeure incertaine (véritables tumuliprotohistoriques, tertres funéraires plus récents, tas d'épierrement, etc.). Il est à noter que la plupart de ces structures se situent en dessous de $1200 \mathrm{~m}$.

La période gallo-romaine est très faiblement représentée sur l'ensemble de la zone (4 sites recensés).

En revanche, les structures attribuées à la période médiévale-moderne sont très nombreuses. Plusieurs groupes ont été distingués, en fonction de la morphologie des structures et des types de regroupements. Enfin, il faut signaler la découverte d'une monnaie d'or, un double écu de Philippe II d'Espagne, dont la présence, dans ce lieu isolé, est surprenante. L'ensemble des données a été intégré dans une base de données, permettant leur analyse par système d'information géographique.

Par ailleurs, des relevés topographiques de structures d'âge historique et des photographies aériennes ont été réalisés (fig. 1 à 3). Il faut signaler également, dans le même cadre géographique, la réalisation d'un master portant sur les burons d'âges moderne et contemporain. (Fig. ${ }^{\circ} 1:$ La montagne de Cauvet : report des sites sur la vue aérienne de ce terroir. Les numéros 17 et 18 sont des tumulide grande taille. Les autres correspondent à des structures médiévales-modernes semi-enterrées), (Fig. n² : SaintClément : report des sites découverts sur la vue aérienne (BD Ortho/IGN) de la partie orientale de la commune.- rond gris: sites préhistoriques;- rond blanc: sites protohistoriques (habitats et tertres présumés);-rond noir:sites de la période médiévale/moderne.) et (Fig. $\mathrm{n}^{\circ} 3$ : Saint-Clément - Belle-Viste-Le Large : report des sites découverts sur la vue aérienne de ce terroir. Il s'agit de structures d'âge médiévalmoderne, souvent rassemblées en « grappes ») 
Dans le domaine des études paléoenvironnementales, il faut signaler l'achèvement des travaux pluridisciplinairesmenés sur le site exceptionnel de la "tourbière de Peyre » (Lacapelle-Barrès), au voisinage duquel se trouve un site du Néolithique ancien. Deux carottages (Peyre 2 et 3) sont venus compléter le prélèvement initial (Peyre 1) et ouvrent des perspectives intéressantes pour la connaissance du paléoenvironnement des périodes récentes (Subboréal et surtout Subatlantique). Une étude palynologique a été conduite sur les niveaux archéologiques du site, sans résultats probants. Les études portant sur la géochimie, la susceptibilité magnétique et le signal paléo-incendie ont été conduites à leur terme, s'accompagnant de la réalisation de six nouvelles ${ }^{14} \mathrm{C}$ destinées à caler les évènements majeurs reconnus.

L'étude du paléo-incendie s'est révélée particulièrement intéressantes. Elle a porté sur 600 échantillons prélevés sur la carotte initiale. La courbe de fréquence des feux met en évidence cinq augmentations significatives de la fréquence des feux qui sont enregistrées au cours de l'Holocène. Leurs maximums respectifs se situent vers 8 600, 6 200, 3 800, 1 800 et $700 \mathrm{cal}$ BC. Les deux plus anciennes s'individualisent avec un bruit de fond important. La première dans le temps, soit vers $8600 \mathrm{cal} \mathrm{BC}$ peut être, sans indice probant d'anthropisation, mise en relation avec la très forte augmentation du régime de feu à la transition Tardiglaciaire-Holocène et précédemment documentée dans d'autres régions d'Europe de l'Ouest. Elle marque un contexte climatique relativement sec ou du moins une saisonnalité contrastée et marquée par une saison sèche annuelle suffisante au déclenchement des feux. Les données de susceptibilité magnétique montrent durant cette période de fortes valeurs et traduisent une contribution détritique importante dans l'accumulation sédimentaire qui explique le bruit de fond dans le signal incendie. Les autres pics peuvent être principalement liés aux pratiques agropastorales, tel que cela a été démontré dans les Pyrénées ou le Centre de la France (Vannière Boris, Galop Didier, Rendu Christine et Davasse Bernard, 2001 - «Feu et pratiques agropastorales dans les Pyréenées orientales: le cas de la montagne d'Enveig, Cerdagne, France», SudOuest Européen, Revue géographique des Pyrénées et du Sud-Ouest,11, p. 29-42 ; Galop Didier, Vannière Boris et Fontugne Michel, 2002 - «Human activities and fires history since $4500 \mathrm{BC}$ on the northern slope of the Pyrenees: a record from Cuguron (central Pyrenees) ", British Archaeological Reports International Series,1063, p. 43-51; Vannière Boris et Martineau Rémi, 2005 - « Histoire des feux et pratiques agraires du Néolithique à l'âge $\mathrm{du}$ Fer en région Centre: implications territoriales, démographiques et environnementales ", Gallia Préhistoire, 47, p.167-186), en accord avec les données fournies par l'analyse pollinique et l'archéologie. L'ensemble des informations permet donc de supposer la présence d'une installation à fonction agro-pastorale au Néolithique ancien, vers $6200 \mathrm{BC}$. C'est la première fois que cette première phase de conquête agropastorale est observée dans le Massif central. Cette phase d'initiation de l'anthropisation est retrouvée dans d'autres massifs montagneux français (Miras Yannick, LaggounDéfarge Fatima, Paul Guenet, Richard Hervé, 2004 - «Multidisciplinary approach to changes in agro-pastoral activities since the Subboreal in the surroundings of the "narse d'Espinasse" (Puy-de-Dôme, French Massif Central) ", Vegetation History and Archaeobotany, 13, p. 91-103), et plus particulièrement le Jura où des indices polliniques d'agriculture ont été datés autour de $5800 \mathrm{cal}$ BC (Richard Hervé, 1994 - "Indices polliniques d'une néolithisation précoce sur le premier plateau du Jura (France)", Compte-rendu de l'académie des sciences de Paris, 318, 2, p. 209-270). Ceci s'inscrit dans un cadre assez large, puisque ces notations de céréales sont interprétées comme des traces d'activités agricoles 
pour d'autres régions d'Europe de l'Ouest (Montagnes : Richard Hervé, 1994 - op. cit.pour le Jura; Haas Jean-Noël, 1996 - Pollen and plant macrofossil evidence of vegetation at Wallisellen-Langachermoos (Switzerlan) during the Mesolithic-Neolithic transition 8500 to 6500 years ago, Berlin, 67 p. pour les Alpes suisses; Zones de basse altitude: Puertas Olivier, 1997 - Évolution de la végétation depuis le Dryas récent dans la plaine littorale de Montpellier (Hérault, France) à partir de l'analyse pollinique. Dynamique naturelle et anthropisation du milieu, thèse, université de Franche-Comté, 337 p.; Riera i Mora Santiago, 1994 - Evolució del paisatge vegetal holocè al pla de Barcelona, a partir de les dades pollíniques, tesi doctoral, universitat de Barcelona, 432 p., etc.).

Enfin, des tests palynologiques ont été effectués sur différents sites tourbeux, disséminés dans la zone d'étude. Si les études menées sur les sites de Peyre-Source (commune de Lacapelle-Barrès) et des sources du Goul (commune de Saint-Clément) ont eu des résultats négatifs, celles conduites sur la tourbière de la Vèze (commune de Pailherols) laissent entrevoir d'intéressantes perspectives pour la réalisation d'une étude pollinique détaillée.

L'année 2007 devrait être consacrée à l'accomplissement de ces travaux et par l'extension géographique de l'ensemble des recherches à la commune voisine de Brezons, dans un cadre géographique marqué par une altitude supérieure.

Yannick Miras et Frédéric Surmely

\section{ANNEXES}


Fig. $\mathrm{n}^{\circ} 1$ : La montagne de Cauvet : report des sites sur la vue aérienne de ce terroir. Les numéros 17 et 18 sont des tumulide grande taille. Les autres correspondent à des structures médiévalesmodernes semi-enterrées

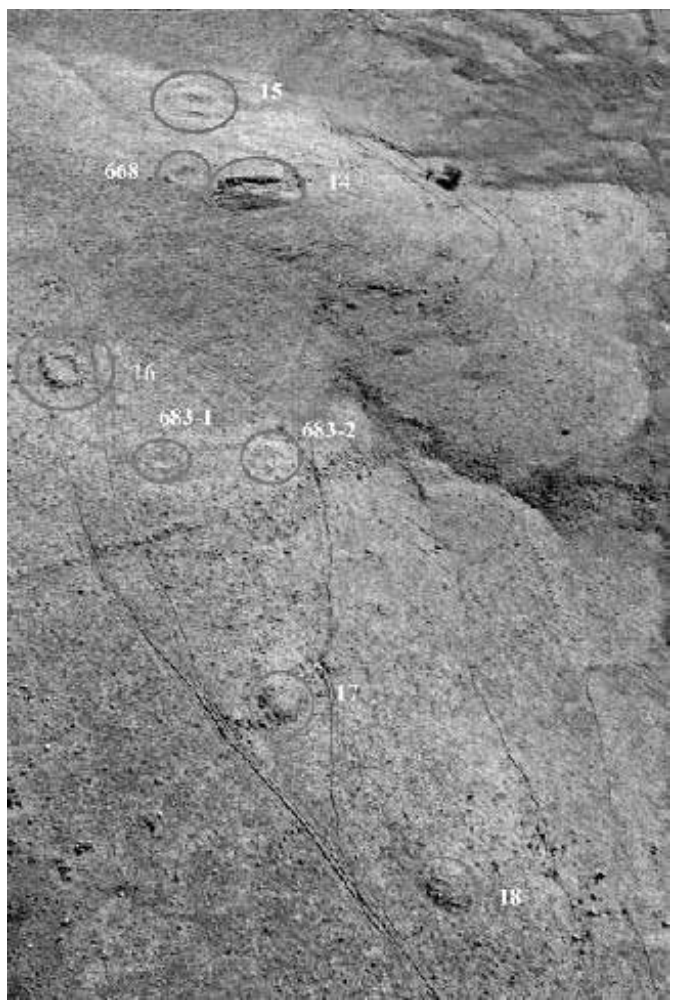

Auteur(s) : Surmely, Frédéric (CNRS). Crédits : Surmely Frédéric, CNRS (2006) 
Fig. $n^{\circ} 2$ : Saint-Clément : report des sites découverts sur la vue aérienne (BD Ortho/IGN) de la partie orientale de la commune.- rond gris : sites préhistoriques ;- rond blanc : sites protohistoriques (habitats et tertres présumés);- rond noir : sites de la période médiévale/ moderne.

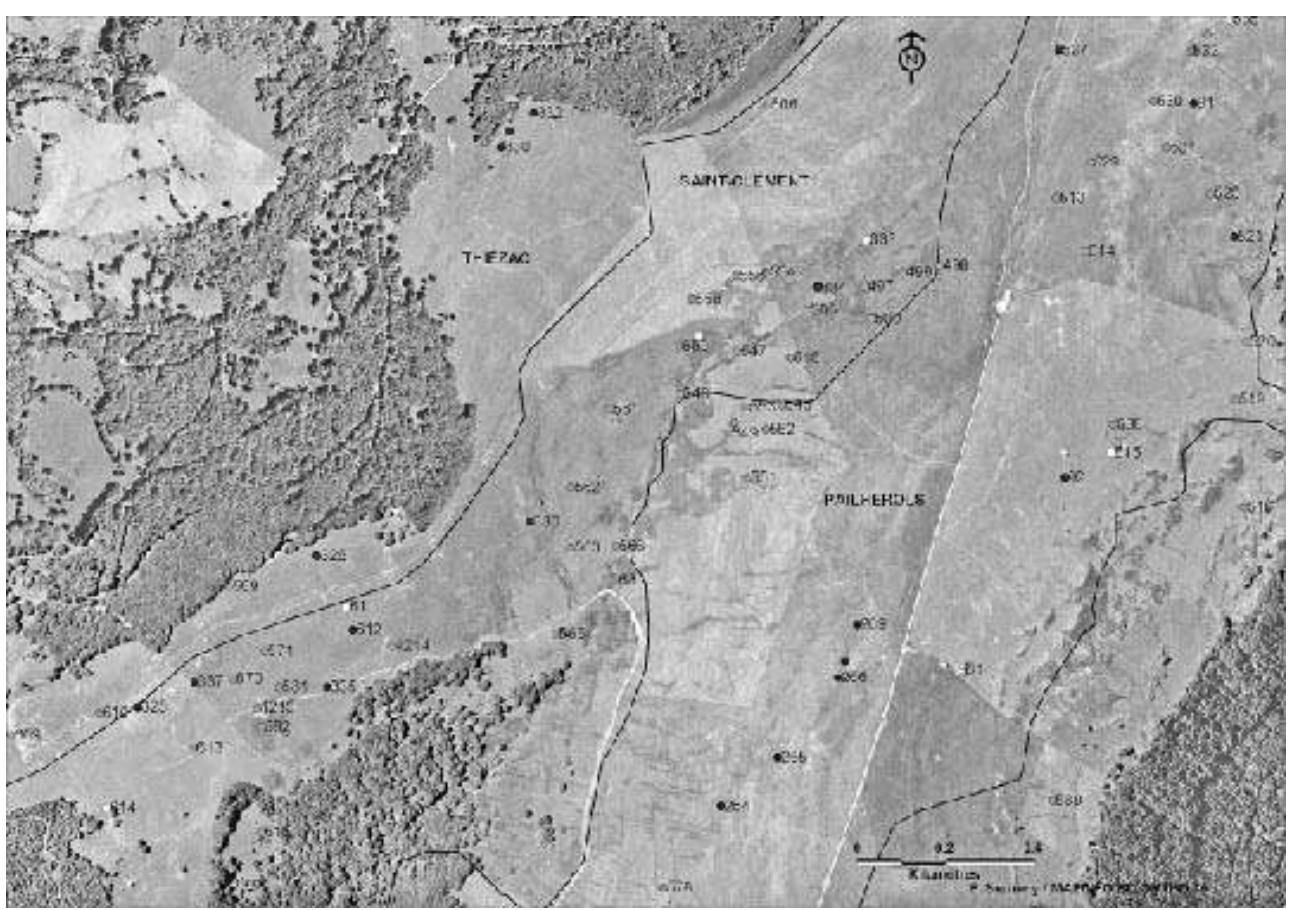

BD Ortho/IGN (2006)

Fig. $n^{\circ} 3$ : Saint-Clément - Belle-Viste-Le Large : report des sites découverts sur la vue aérienne de ce terroir. Il s'agit de structures d'âge médiéval-moderne, souvent rassemblées en " grappes "

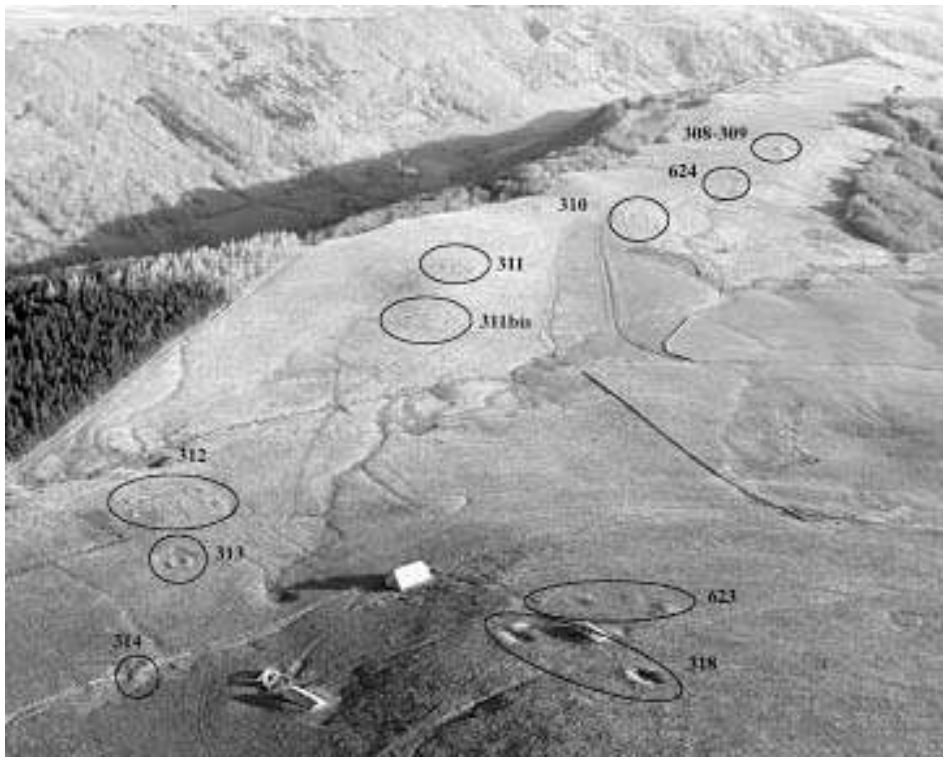

Auteur(s) : Surmely, Frédéric (CNRS). Crédits : Surmely Frédéric, CNRS (2006) 
INDEX

Index géographique : Auvergne, Allier (03), Saint-Clément, Lacapelle-Barrès, Malbo, Pailherols Thèmes : agriculture, base de données, céréale, coffre en pierre, incendie, industrie lithique, monnaie, paléoenvironnement, palynologie, pastoralisme, photographie aérienne, radiocarbone, SIG, tertre funéraire, tourbière

Index chronologique : âge du Bronze, âge du Fer, Empire romain, ép. contemporaine, Mésolithique, Moyen Âge, Néolithique, Temps Modernes

operation Prospection thématique (PRT)

\section{AUTEURS}

\section{YANNICK MIRAS}

BEN

FRÉDÉRIC SURMELY

CNRS 